\title{
Biologists create more precise molecular scissors for genome editing
}

Engineered Cas9 enzyme makes fewer mistakes.

Heidi Ledford

01 December 2015

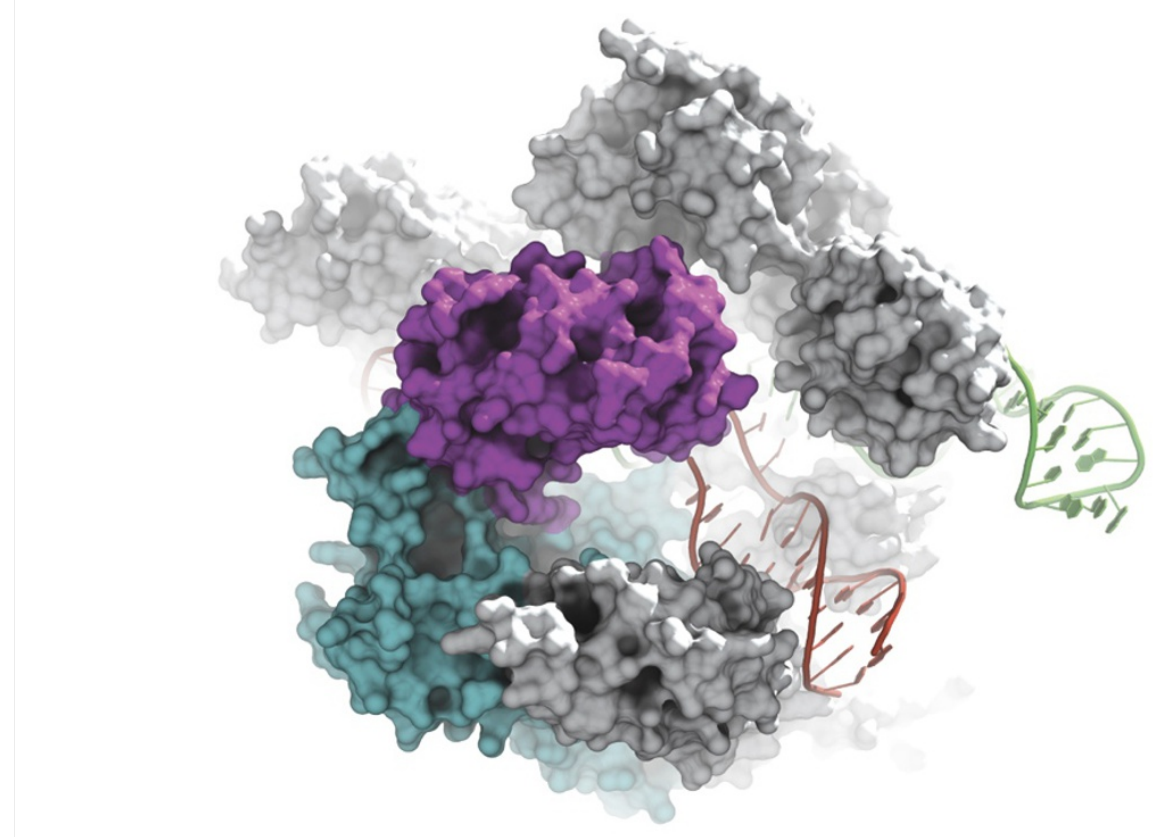

lan Slaymaker, Broad Institute

The Cas9 enzyme cuts DNA (in red) to which it is guided by an RNA sequence (in green).

By tweaking an enzyme that cuts DNA, synthetic biologists say that they can make genome editing even more specific — an essential improvement if the technique is to be deployed in the clinic to treat genetic diseases.

The enzyme, called Cas9, is a key component of a molecular-editing system that enables researchers to alter particular DNA sequences in the genome. That technology, called CRISPR-Cas9, is so quick, cheap and easy to use that it is already changing how genetic research is done and could one day provide a way to correct genetic mutations that cause disease in humans. On 1 December, researchers from the United States, the United Kingdom and China are convening in Washington DC to discuss the ethics and applications of gene-editing in humans.

But before any clinical applications, patients and regulators must be sure that the Cas 9 enzyme will not cause dangerous off-target damage to the genome. "For therapeutic applications, we all want to proceed with maximum caution to make sure we're not modifying the genome at undesired locations," says David Liu, a chemical biologist at Harvard University in Cambridge, Massachusetts.

\section{Error rate cuts}

Researchers have already been tweaking the components of CRISPR-Cas9 to drive down its error rate. They have tweaked the RNAs that guide the Cas 9 enzyme to a specific site in the genome ${ }^{1}$, for example, and engineered the system so that researchers can easily switch it off, so that the enzyme does not have as much opportunity to make unwanted changes 2,3 .

Synthetic biologist Feng Zhang at the Broad Institute of MIT and Harvard in Cambridge decided to focus on engineering the Cas9 enzyme itself. He and his team altered the enzyme so that it is less likely to act at sites with mismatches between the RNA that guides the enzyme and the DNA that it targets. They generated several versions of Cas 9 that reduced off-target errors at least tenfold compared with unaltered Cas 9 enzymes ${ }^{4}$. 
"It's a matter of tempering the excess 'enthusiasm' of Cas9, so that it grudgingly accepts on-target sequences and nothing else," says Liu, who was not involved in the work.

Zhang's team have created three new versions of Cas9 that, they report, made fewer mistakes but were just as active at their target sites as ordinary Cas9. And Zhang notes that unlike other ways to reduce CRISPR-Cas9 error rates, the new enzymes require no changes to the CRISPR-Cas9 protocol already used by many researchers.

\section{More than one answer}

For many research applications, an unaltered Cas9 enzyme will do, says Liu. But for therapies, researchers would like to drive down the error rate so that it is no higher than the normal rate of DNA mutation in human cells. On its own, the tenfold improvement that the engineered enzyme achieves isn't quite enough, Liu estimates; he thinks that for therapies, the error-rate of CRISPR-Cas9 gene editing needs to be made several orders of magnitude more specific. But combining various methods for improving specificity might well do the trick, he says.

Zhang compares the problem to trying to make a car go faster: the new Cas9 enzyme is like putting in a bigger engine. "This can still be combined with other modifications, like adding a spoiler, leading to a souped-up enzyme," he says. "There are many ways to increase speed."

Nature I doi:10.1038/nature.2015.18932

\section{References}

1. Fu, Y., Sander, J. D., Reyon, D., Cascio, V. M. \& Joung, J. K. Nature Biotechnol. 32, 279-284 (2014).

2. Zetsche, B., Volz, S. E. \& Zhang, F. Nature Biotechnol. 33, 139-142 (2015).

3. Davis, K. M., Pattanayak, V., Thompson, D. B., Zuris, J. A. \& Liu, D. R. Nature Chem. Biol. 11, $316-318$ (2015).

4. Slaymaker, I. M. et al. Science http://dx.doi.org/10.1126/science.aad5227 (2015). 